\title{
Lidil
}

Revue de linguistique et de didactique des langues

49 | 2014

L'analyse des données didactiques

\section{La littérature au secondaire québécois : conceptions d'enseignants et pratiques déclarées en classe de français}

Literature in Quebec Secondary Schools: Conceptions of Teachers and Declared Practices and Methods in Teaching in French Classes

\section{Judith Émery-Bruneau}

\section{OpenEdition}

\section{Journals}

Édition électronique

URL : http://journals.openedition.org/lidil/3454

DOI : 10.4000/lidil.3454

ISSN : $1960-6052$

Éditeur

UGA Éditions/Université Grenoble Alpes

Édition imprimée

Date de publication : 25 mai 2014

Pagination : 71-91

ISBN : 978-2-84310-272-1

ISSN : $1146-6480$

\section{Référence électronique}

Judith Émery-Bruneau, « La littérature au secondaire québécois : conceptions d'enseignants et pratiques déclarées en classe de français », Lidil [En ligne], 49 | 2014, mis en ligne le 25 novembre 2015, consulté le 19 avril 2019. URL : http://journals.openedition.org/lidil/3454 ; DOI : 10.4000/ lidil.3454 


\title{
La littérature au secondaire québécois : conceptions d'enseignants et pratiques déclarées en classe de français
}

\author{
Judith Émery-Bruneau*
}

\begin{abstract}
RÉSUMÉ
L'objectif de cette étude est de décrire les conceptions et les pratiques d'enseignement de la littérature en classe de français au secondaire québécois. À cette fin, nous avons réalisé des entretiens semi-dirigés auprès de dix enseignants et filmé des séquences d'enseignement de la littérature. Cet article analyse les contenus des entretiens relatifs aux conceptions et aux pratiques déclarées de ces enseignants. Cinq aspects dominants de leur rapport à la littérature sont décrits plus finement : leurs conceptions de la littérature, leurs critères de littérarité, les œuvres enseignées, les finalités de l'enseignement de la littérature et le rôle de la littérature dans le développement des savoirs. Dans la majorité des cas, le rapport des enseignants à la littérature est influencé par leurs pratiques personnelles de la littérature, leur formation initiale et surtout le contexte d'enseignement.
\end{abstract}

\section{ABSTRACT}

The aim of this study describes the conceptions of French teachers in Quebec secondary schools, their declared practices and methods in teaching literature. Semi-structured interviews have been conducted with ten teachers and fews sequences of teaching literature have been filmed. The content analysis of these interviews with regard to the conceptions and practices declared by the teachers will be presented here. Four aspects of their relation to culture are described: the conceptions of literature, the corpus taught, the goal of teaching literature and its role in the development of knowledge. In the majority of cases, the relation of the teachers to literature is influenced by their personal practices of literature, their initial training and above all, the teaching context.

* Université du Québec en Outaouais. 
Au Québec, le ministère de l'Éducation a procédé à la révision des programmes scolaires pour l'enseignement du français au secondaire à quatre reprises au cours des 45 dernières années (1969, 1980, 1995, 2005/2009). Depuis 1995, les prescriptions concernant l'enseignement des textes littéraires donnent de plus en plus de place à un contenu littéraire, particulièrement au $2^{\mathrm{e}}$ cycle du secondaire. Même si le programme actuel contient une section intitulée «notions et concepts de littérature» (MÉLS, 2009, p. 146-159), les savoirs littéraires à enseigner demeurent flous, tout comme le corpus d'œuvres qui n'est marqué que d'une quantité (cinq œuvres), à répartir en genres et auteurs variés. Puisque les enseignants sont libres de choisir les œuvres à l'étude, les modes de lecture (lecture stratégique, esthétique, littéraire, etc.), les approches (constructiviste, cognitiviste, etc.), les démarches (inductive, déductive, etc.) et les activités d'évaluation (examen de compréhension de lecture, production d'une analyse littéraire, cercle de lecture, etc.) à privilégier, leurs choix des œuvres littéraires à enseigner s'appuient, selon Dezutter (2007), sur leurs connaissances, leurs gouts, leurs objectifs pédagogiques ou les contraintes matérielles du milieu. Outre ce qui concerne le choix des corpus, on ignore encore quels sont les objets enseignés sous l'appellation «littérature» dans les classes de français du secondaire québécois et comment ces objets sont enseignés.

Des recherches descriptives ont été menées sur les objets enseignés et les pratiques d'enseignement au secondaire québécois pour décrire comment l'écriture et la grammaire (Chartrand \& Lord, 2010, 2013), la communication orale (Sénéchal \& Chartrand, 2012) et la culture (Falardeau \& Simard, 2011) sont enseignées au secondaire, mais aucune recherche n'a encore étudiée spécifiquement comment la littérature est enseignée au secondaire québécois. La situation n'est pas unique au Québec : Daunay soulignait en 2007 que «dans le domaine de l'enseignement de la littérature, l'analyse des pratiques enseignantes demeure encore peu développée» (p. 157). Or, nous supposons que les pratiques d'enseignement liées à cet objet sont aussi hétéroclites que les conceptions rangées sous l'étiquette «littérature» sont polysémiques. Qu'est-ce qu'enseigner la littérature pour les enseignants de français? S'agit-il de faire lire un corpus de textes? D'auteurs à connaitre? D'une manière d'écrire, de lire, de dire ou d'entendre? De visions du monde? Enseigne-t-on la «littérature», à la fois comme pratique et comme objet? Bref, comment les enseignants conçoivent-ils et enseignent-ils cet objet? 
Approfondir cette question est le défi que s'est donné l'équipe de RELIT (Recherches sur l'Enseignement de la LITtérature) ${ }^{1}$.

\section{Littérature et rapport à la littérature : éléments du cadre théorique}

S'inscrivant dans une perspective didactique, l'équipe de RELIT définit la littérature comme un système composé de productions verbales visant à travailler le langage en tant qu'objet esthétique et de pratiques langagières qui les rendent possible. Nous sommes d'avis que la pratique de la littérature

n'est pas seulement un support de lecture et de connaissances, mais [c'est] aussi l'objet d'une diversité d'activités d'écriture et d'oralité, les unes à dimension créative ou «hypertextuelle» (production d'autres textes ou performances oralisées au départ des textes lus), les autres à dimension critique ou «métatextuelle» (commentaires oraux et écrits)

(Simard, Dufays, Dolz \& Garcia-Debanc, 2010, p. 334).

La littérature, en plus d'être un objet à lire, à écrire, à dire et à entendre, est aussi un objet à connaitre et à penser, objet qui doit par conséquent être vu dans toutes ces dimensions pour être en mesure de faire une analyse fine de la façon dont on la pratique ou l'enseigne. Notre recherche s'intéresse de près à l'enseignement des textes littéraires, ce qui désigne toute production verbale, écrite ou orale, visant à travailler le langage, car la littérature «en tant qu'art du verbe [...] explore les possibilités de dire le monde, de le représenter et même de le reconstruire par le langage» (Simard, 1997a, p. 56). À l'instar de Dufays, Lisse \& Meurée (2009), on peut dès lors qualifier un texte de «littéraire» à l'aide de critères internes relevant de la forme (stylistique), des contenus (thématiques, valeurs exprimant une vision du monde, etc.) et des liens entre les textes (intertextualité, transtextualité, etc.) et de critères externes relevant de l'auteur (contexte sociohistorique de production), du milieu social (champs de production et institution littéraire) et du lecteur

1. L'équipe de RELIT a reçu une subvention du Fonds québécois de la recherche sur la société et la culture (FQRSC), de 2012 à 2015. Cette contribution présente l'analyse des résultats de la phase 1 de RELIT (conceptions et pratiques déclarées). Les résultats de la phase 2, portant sur les pratiques effectives de l'enseignement de la littérature, seront présentés dans une prochaine contribution. 
(compréhension/interprétation, expérience affective, etc.). La littérature est donc un système complexe composé de l'ensemble de ces critères complémentaires. Dans cette perspective, s'intéresser à la littérature comme objet enseigné oblige à prendre en compte des productions littéraires (écrites ou orales), des pratiques de la littérature (de lecture, d'écriture et d'oralité) et des connaissances littéraires enseignées en classe de français.

Pour comprendre la littérature en tant qu'objet enseigné, nous avons choisi de l'étudier en tenant compte du rapport de l'enseignant à l'objet enseigné. Ainsi, pour étudier les liens entre les conceptions qu'ont les enseignants de la littérature et leurs pratiques déclarées, nous analysons leur rapport à la littérature. Cette notion de «rapport à» est développée dans la continuité des travaux sur le rapport au savoir (Charlot, Bautier \& Rochex, 1992), le rapport à l'écriture (Barré De Miniac, 2000), le rapport à l'écrit (Blaser, 2007, 2009; Chartrand \& Blaser, 2008), le rapport à la culture (Falardeau \& Simard, 2011) et le rapport à la lecture littéraire (Émery-Bruneau, 2010, 2011, 2012). Nous définissons le rapport à la littérature (désormais RL) comme l'ensemble diversifié de relations dynamiques d'un sujet avec les productions littéraires et les pratiques de la littérature. L'analyse du RL se centre à la fois sur l'objet (textes littéraires, savoirs littéraires, etc.) et sur le sujet (conceptions et pratiques de la littérature, lesquelles se construisent et se transforment dans chaque activité de production ou de réception de textes littéraires).

Puisque nous étudions le RL d'enseignants, notre modèle est organisé en deux plans : le plan personnel et le plan didactique (ÉmeryBruneau, 2010). Le plan personnel est la façon dont un sujet pratique ou donne sens à la littérature alors que le plan didactique est la façon dont l'enseignant interprète le RL des élèves et la façon dont il entend le transformer en décomposant l'objet «littérature» en éléments enseignables. Sur la base de nos travaux antérieurs, chacun de ces deux plans est composé des dimensions subjective, sociale, épistémique et praxéologique, lesquelles se déplient en plusieurs aspects ${ }^{2}$ qui s'interconnectent, s'imbriquent, se complètent et sont (inter)actifs. Précisons qu'en continuité de nos travaux sur le rapport à la lecture littéraire (Émery-Bruneau, 2010, 2011, 2012), centrés sur une des pratiques de la littérature, soit la lecture littéraire, le RL est composé de ces mêmes

2. Ceux-ci sont présentés dans la deuxième partie du texte où nous décrivons la méthodologie, et sont définis dans les tableaux en annexes. 
quatre dimensions, lesquelles ont toutefois été redéfinies de façon à pouvoir analyser l'ensemble des pratiques de la littérature (non seulement la lecture, mais aussi les pratiques d'écriture et d'oralité) et des productions littéraires qui s'y rattachent. Par exemple, la dimension praxéologique $d u$ plan personnel $d u R L$ concerne «les pratiques de la littérature, les activités concrètes de lecture, d'écriture ou d'oral (à dimension créative, analytique ou appréciative) qui orientent les pratiques ou les réflexions du sujet à l'égard de la littérature.» Présentées dans la grille d'analyse de contenu en annexe, les composantes de cette dimension sont : types de production ou objet de réception (quoi?); lieux de production ou de réception (où?); contextes de production ou de réception (quand?); objectifs de production ou de réception (pourquoi ?); modes de production ou de réception (comment?); temps et fréquence de production ou de réception (combien?); moyens didactiques ou pédagogiques mis en œuvre par exemple, par les enseignants préuniversitaires, dans les pratiques de littérature. Nous l'avons dit, cette définition a été développée à partir de nos travaux précédents sur la lecture littéraire (Émery-Bruneau, 2010) où la dimension praxéologique du plan personnel du rapport à la lecture littéraire concerne les pratiques de lecture littéraire, c'est-à-dire les activités concrètes qui orientent la pratique ou la réflexion du sujet-lecteur. Les composantes de cette dimension du rapport à la lecture littéraire, moins englobantes que celles du RL, sont strictement tournées vers la réception du sujetlecteur : types de textes lus; lieux de lecture; contexte de lecture; objectifs de lecture; réception du texte et construction du texte singulier; temps et fréquence de lecture; moyens didactiques ou pédagogiques mis en œuvre dans les activités de lecture. Voilà comment les dimensions du rapport à la littérature (RL) et leurs composantes ont été définies par l'équipe de RELIT et en quoi elles se distinguent de celles du rapport à la lecture littéraire.

\section{Méthodologie}

Issue des travaux menés dans le cadre de RELIT, notre étude vise ici à décrire les conceptions et les pratiques déclarées menées sous l'appellation «enseignement de la littérature» en classe de français au secondaire québécois pour comprendre les effets du RL sur la façon dont cet objet est enseigné. Les objectifs spécifiques de la phase 1 de cette recherche, et dont cette contribution fait l'objet, sont les suivants : 1) décrire le RL d'enseignants de français du secondaire; 2) décrire leurs pratiques 
déclarées, menées sous l'appellation «enseignement de la littérature»; 3 ) analyser les relations entre leur RL et leurs pratiques déclarées.

Cette recherche descriptive de type qualitatif se situe au carrefour de courants de recherche en didactique du français croisant l'analyse du RL d'enseignants de français et la description d'un objet enseigné (littérature). Pour atteindre nos objectifs, nous avons suivi dix enseignants de français du secondaire $(\mathrm{n}=10)$ participant sur une base volontaire, dont quatre enseignent au $1^{\text {er }}$ cycle $^{3}$ (Julie, Sébastien, Sophie, Guylaine) et six enseignent au $2^{\mathrm{e}}$ cycle $^{4}$ (Stéphanie, Fabrice, Sonia, Cindy, Monique, Gilles). Différentes variables les distinguent, dont leur formation initiale, leurs expériences, leur âge et leur contexte d'enseignement.

Pour comprendre le RL de ces enseignants, un entretien individuel semi-dirigé (d'une durée de 75 minutes) a permis de recueillir leurs conceptions de la littérature et de son enseignement. L'entretien les amenait de surcroit à décrire leurs pratiques, à verbaliser leurs intentions didactiques orientant leurs séquences d'enseignement de la littérature et à expliquer la place et le rôle qu'occupent ces séquences d'enseignement dans leur planification annuelle. Le guide d'entretien était composé de six questions ouvertes ${ }^{5}$ et de quelques sous-questions auxquelles recourait la chercheuse si l'enseignant demandait des précisions, était à court d'exemples ou ne répondait pas directement à la question, entre autres.

Transcrits sous forme de verbatim, les entretiens ont fait l'objet d'une analyse de contenu (L'Écuyer, 1990) avec une grille d'analyse conçue à partir du cadre théorique du RL. Sous les deux plans et les quatre dimensions du RL, 46 catégories ont été utilisées pour coder les énoncés des entretiens (voir tableaux en annexe). Comme nous l'avons expliqué plus haut avec l'exemple de la dimension praxéologique, la plupart de ces 46 catégories ont été formulées à partir de nos travaux antérieurs

3. Le $1^{\text {er }}$ cycle comprend les $1^{\text {re }}$ et $2^{\mathrm{e}}$ secondaires. Les élèves sont généralement âgés de 12 à 14 ans.

4. Le $2^{\mathrm{e}}$ cycle comprend les $3^{\mathrm{e}}, 4^{\mathrm{e}}$ et $5^{\mathrm{e}}$ secondaires. Les élèves sont généralement âgés de 14 à 17 ans.

5. Par exemple : «Pourriez-vous me parler de littérature et me dire ce que vous entendez par "littérature"?» (question 1); «Pourriez-vous me décrire des cours ou des ensembles de cours de votre planification annuelle qui portent sur la littérature et qui sont représentatifs de votre façon de l'enseigner dans votre classe de français?» (question 3); «Pour quelle raison croyez-vous qu'il soit pertinent d'enseigner la littérature en classe de français au secondaire? Qu'en pensent les élèves, à votre avis?» (question 5). 
sur le rapport à la lecture littéraire (Émery-Bruneau, 2010, 2011, 2012), mais elles ont toutes été redéfinies de façon à y inclure l'ensemble des pratiques de la littérature, c'est-à-dire non seulement celles de la lecture littéraire, mais aussi celles de l'écriture et de l'oralité. Au cours de la première analyse des entretiens, des catégories émergentes ${ }^{6}$ se sont ajoutées aux catégories préétablies. Une deuxième analyse a donc été réalisée sur l'ensemble des données afin de nous assurer que tous les énoncés figuraient à l'intérieur des catégories appropriées. C'est lors de la deuxième analyse qu'un accord intercodeurs a été réalisé : le pourcentage d'accord pour le découpage des énoncés et leur codification s'élevait à $90 \%$. Une troisième et dernière vérification des données a finalement permis de valider l'analyse de contenu.

\section{Conceptions et pratiques déclarées : les tendances émergentes}

Comme les données recueillies lors des entretiens semi-dirigés sont nombreuses, nous avons choisi de n'en présenter qu'une partie, représentative de l'ensemble. Il s'agit des catégories qui ont trouvé le plus grand nombre d'énoncés et qui permettent de saisir davantage les conceptions des sujets. Nous rendons compte des résultats de six catégories, appartenant à trois des quatre dimensions du RL :

- représentations de la littérature et critères de littérarité (dimension subjective);

- types de production ou objets de réception de la littérature (dimension praxéologique), ce qui, dans les données recueillies, renvoie surtout aux œuvres enseignées;

- finalités ou objectifs de production ou de réception de la littérature (dimension praxéologique);

6. Voici les huit catégories qui ont émergé lors de la première analyse, qui ont été ajoutées aux catégories préétablies et qui ont été utilisées lors de la deuxième analyse de contenu : Critères de littérarité des textes (PPDSu-6); Critères de sélection ou valeur symbolique des savoirs (PPDE-5); Rôle des enseignants préuniversitaires (PPDSo-4); Rôle de la formation initiale (PPDSo-5); Rôle de la formation continue (PPDSo-6); Critères des élèves quant à la littérarité des textes (PDDSu-6); Critères de sélection ou valeur symbolique des savoirs des élèves (PDDE-5); Rôle des enseignants du primaire ou du secondaire (PPDSo-4). 
- rôle de la littérature dans le développement des savoirs (dimension épistémique).

L'analyse de ces aspects et des interactions entre les dimensions du RL des sujets est présentée et discutée dans les quatre sous-sections qui suivent.

\subsection{Des conceptions de la littérature marquées par les pratiques de littérature et par la formation initiale}

Les réponses à la première question posée afin de faire émerger les conceptions de la littérature ont fait ressortir une vision dichotomique de la littérature qui domine chez 7 des 10 enseignants interrogés. Ces derniers partagent la littérature en deux types : la «grande littérature» et la «petite littérature», classement qui rappelle le modèle sociologique de la littérature (Bourdieu, 1992) où le champ littéraire est composé des sphères de production restreinte et large. Ainsi définissent-ils la «grande littérature» comme les canons littéraires (Fabrice, Julie et Sophie) au sens de classiques de la littérature, textes nécessitant une réflexion (Guylaine) et un travail intellectuel du lecteur et permettant de voir le monde dans une perspective différente (Cindy) de celle à laquelle le lecteur est habitué. En opposition à la «grande littérature», la «petite littérature» est, pour eux, celle qui divertit et qui permet de se détendre (Cindy et Guylaine), qui exige peu ou pas d'efforts intellectuels, qui demeure superficielle (Guylaine, Stéphanie, Sonia, Cindy). Par exemple, Stéphanie compare la «grande littérature» à des vêtements de haute couture, indémodables, et à de la fine gastronomie, et la "petite littérature» à du fastfood. Les œuvres inscrites dans le champ de production large manqueraient donc de substance selon la majorité des enseignants interrogés, bien qu'on en retrouve dans les corpus enseignés, comme nous le verrons.

Lorsqu'il leur a été demandé de préciser leurs conceptions en nommant des critères ${ }^{7}$ pour les expliciter, nous avons constaté que leur vision est influencée par leurs pratiques de la littérature et par leur formation en études littéraires. Voici différents exemples. Un enseignant en insertion

7. Il s'agit de critères de littérarité que les sujets ont mentionnés sans forcément recourir au métalangage littéraire. Lors du traitement des données, leur propos ont été analysés à l'aide du classement de Dufays, Lisse et Meurée (2009). Les catégories de ces critères - internes et externes - sont décrits dans la section 1, qui présente les principaux éléments de notre cadre théorique. 
professionnelle (Sébastien), qui se dit aussi non lecteur, pense que tous les textes écrits dans le but de divertir peuvent être considérés comme littéraires : ses critères de littérarité sont peu nombreux et semblent surtout tournés vers la réception affective. Ayant un propos plus précis, Sophie affirme que pour être considérés comme littéraires, les textes doivent absolument émouvoir le lecteur, par exemple, en lui permettant de vivre une catharsis, d'être confronté à une nouvelle vision du monde ou de vivre des émotions fortes (pleurer, être en colère, etc.) : ses critères de littérarité intègrent surtout la réception affective et l'intention de l'auteur. Précisons que Sophie, en plus d'avoir récemment complété un certificat en création littéraire, écrit de la poésie tous les jours depuis plusieurs années et performe dans des joutes de slam depuis au moins six ans : son RL est sans doute marqué par ses pratiques d'écriture créative et de performances slamées où les émotions sont au cœur de la joute. Gilles, un enseignant âgé de 60 ans et diplômé en études littéraires, se décrit comme un «très grand lecteur [ayant] lu des milliers de livres, un homme sensible, facilement ému, voire ébranlé à la lecture d'une œuvre». Pour lui, la littérature est une véritable expérience «humaine» (pour mieux se comprendre), «philosophique» (pour mieux comprendre le monde) et «physique» (tous ses sens sont convoqués dans l'expérience littéraire). Ces trois enseignants se sont surtout appuyés sur des critères externes pour définir la littérature : la réception affective du lecteur et l'intention de l'auteur.

Par ailleurs, la littérature est aussi vue comme un objet esthétique, lequel est manifestement un texte écrit reconnu. Cinq enseignants partageant cette conception (Julie, Stéphanie, Cindy, Sonia, Fabrice) disent que la littérature est constituée d'auteurs reconnus dont les œuvres rencontrent deux critères de littérarité : 1) l'esthétisme, décrit notamment comme une «exploration des limites du langage, des jeux de sémantique, des associations insolites» (Fabrice); 2) la vision du monde de l'auteur, caractérisée par «un style lui permettant de traiter un sujet sous un angle original» (Cindy). Pour ces cinq enseignants, la littérarité renvoie principalement aux critères internes, dont le style, ainsi qu'à des critères externes, parmi lesquels on retrouve la vision du monde et de l'auteur, et la reconnaissance de l'institution littéraire.

Deux grandes tendances s'observent: d'une part, ceux qui voient la littérature comme une expérience surtout affective (Sébastien, Sophie, Gilles), marquée d'émotions et de compréhension de soi, d'autre part, ceux qui la voient comme un ensoi objectif (Fabrice, Cindy, Julie, Stéphanie, Sonia), marqué d'un travail sur la langue, d'une histoire et d'une 
vision du monde, et étudié pour construire des connaissances. En outre, nos observations font ressortir un lien étroit entre la formation reçue et les conceptions de la littérature : les enseignants ayant reçu une formation plus élaborée en études littéraires ${ }^{8}$ (Fabrice, Sonia, Gilles, Sophie) ont donné beaucoup plus d'exemples de leurs pratiques de littérature, ont convoqué plus de critères pour définir la littérature et semblent en avoir une vision plus complexe.

\subsection{Cuuvres enseignées : choix affectifs et contraintes matérielles}

Pendant l'entretien, les enseignants ont été invités à nommer les œuvres qu'ils mettent à l'étude. Nous les avons aussi amenés à parler de la façon dont ils introduisent celles-ci dans leur planification de cours. Nos résultats vont dans le même sens que ceux de Dezutter (2007) qui notait une très grande diversité dans la sélection des corpus de lecture des élèves, car pour les 10 enseignants de notre étude, 74 titres et 69 auteurs différents ont été nommés. Les auteurs français (32 occurrences) et québécois (20 occurrences) ont la cote, suivis par les auteurs américains traduits (8 occurrences). De cette grande diversité ressortent néanmoins trois tendances.

La première tendance porte sur la préséance du genre romanesque. Très peu de pièces de théâtre sont travaillées ${ }^{9}$ et aucun recueil de poésie n'est à l'étude. Bien que les enseignants soient contraints d'enseigner cinq œuvres littéraires par année et que la majorité doive être à dominante narrative, leur choix du sous-genre romanesque ${ }^{10}$ se base plus sur leurs intérêts personnels ou choix didactiques que sur les prescriptions. Par exemple, le roman policier, pourtant proposé pour la $2^{\mathrm{e}}$ secondaire, est enseigné par des enseignants de $5^{\mathrm{e}}$ secondaire qui considèrent que les élèves seront plus mûrs pour être plongés dans cet univers plutôt noir et terrifiant.

8. Ce qui veut dire que leur programme de formation en enseignement était composé d'une trentaine de crédits en études littéraires ou qu'ils ont complété une formation en lettres.

9. Le théâtre est prescrit au programme de la $4^{\mathrm{e}}$ secondaire (et l'était aussi dans les programmes de 1995 et de 1980). Il s'agit toutefois de la seule des cinq années scolaires où nous n'avons pas pu interroger d'enseignants, faute de volontaires.

10. Par exemple, le roman historique, policier, d'aventures ou fantastique. 
La deuxième tendance met en lumière des distinctions nettes entre les $1^{\text {er }}$ et $2^{\mathrm{e}}$ cycles. La majorité des œuvres à l'étude au $1^{\text {er }}$ cycle relève de la littérature de jeunesse, surtout d'origine québécoise. Au $2^{\mathrm{e}}$ cycle, les corpus sont partagés entre des classiques de la littérature et des œuvres contemporaines appartenant à la sphère de production large (Bourdieu, 1992). Ces choix vont dans le même sens que les finalités de l'enseignement des textes littéraires qu'ils privilégient (et qui fait l'objet de la partie suivante). Au $1^{\text {er }}$ cycle, les enseignants mettent surtout l'accent sur le gout de lire et sur le développement de l'identité personnelle et de lecteur des élèves. Ils travaillent surtout la compréhension en lecture à partir de textes proches de la culture première des élèves. Au $2^{\mathrm{e}}$ cycle, des questions plus complexes, souvent d'ordre philosophique ou social, sont creusées à l'aide des œuvres proposées, et les enseignants travaillent par la même occasion l'argumentation, objet au cœur de l'examen certificatif ministériel.

La troisième tendance fait ressortir deux critères pour choisir des œuvres : le premier concerne des textes ou des auteurs que les enseignants affectionnent particulièrement et qu'ils veulent faire connaitre et le deuxième concerne les contraintes matérielles (travailler avec des séries-classes achetées par l'école). Cette tendance était aussi apparue dans l'enquête de Dezutter (2007). Pour le premier critère, au moins quatre enseignants affirment préférer enseigner des œuvres qu'ils aiment, car ils peuvent alors transmettre leur passion et espèrent ainsi motiver leurs élèves. Pour le deuxième critère, comme les enseignants des écoles publiques sont contraints par la loi sur l'instruction publique qui n'autorise pas les enseignants à imposer l'achat de livres par les parents, leur institution achète des séries de livres (par exemple 30 copies de L'Étranger) et les élèves les lisent en classe ${ }^{11}$. Les séries étant en nombre limité et insuffisant pour couvrir les exigences du programme, la très grande majorité des enseignants propose des œuvres au choix, lesquelles ne sont pas travaillées en classe, mais font tout de même l'objet d'une évaluation. D'ailleurs, tous les enseignants affirment rattacher systématiquement au moins une activité d'évaluation à chaque œuvre du corpus (examen de compréhension de lecture, production d'un résumé ou d'une analyse littéraire, etc.), même si les œuvres n'ont pas

11. Leurs fonds étant limités, les écoles ne peuvent ni renouveler les sériesclasses tous les ans ni acheter au moins cinq titres différents pour couvrir une année scolaire (à moins de ne pas acheter 30 copies du même titre, mais plutôt six copies de cinq titres différents). 
fait l'objet d'activités d'enseignement et d'apprentissage en classe. Ces contraintes matérielles entrainent au moins deux problèmes : 1) les enseignants peinent à respecter les prescriptions;2) ils ne se trouvent pas dans des conditions qui leur permettent de travailler les œuvres intégrales comme le recommandent les recherches en didactique de la littérature depuis plus de 20 ans (Biard \& Denis, 1993; Veck, 1998; Langlade, 2002).

\subsection{Finalités de l'enseignement de la littérature : tensions entre les cycles, les classes régulières et les programmes d'élite}

Les conceptions qu'ont les enseignants de la littérature (dimension subjective du plan personnel du RL) se traduisent dans les corpus et les finalités de son enseignement (dimension praxéologique du plan didactique du RL). Une division très claire apparait entre les finalités poursuivies au $1^{\text {er }}$ cycle, plutôt d'ordre psychoaffectif, et celles du $2^{\mathrm{e}}$ cycle, plutôt d'ordre esthétique, culturel et philosophique (Simard, 1997a). Tous les enseignants du $1^{\text {er }}$ cycle disent enseigner la littérature davantage dans le but de leur faire aimer la lecture et de développer leur compétence en lecture; les enseignants du $2^{\mathrm{e}}$ cycle désirent surtout développer le bagage culturel de leurs élèves ou les amener à réfléchir sur le monde. Cet important clivage entre les choix pédagogiques et didactiques des enseignants du $1^{\text {er }}$ et du $2^{\mathrm{e}}$ cycle n'est pas sans rappeler les travaux de De Beaudrap (2004) où, dans son étude sur les représentations de la littérature et de son enseignement menée auprès de 600 professeurs stagiaires, une tension très forte apparait à l'égard de leur identité professionnelle : l'enseignant du collège (équivalent au $1^{\text {er }}$ cycle québécois) se considère comme un enseignant de français; l'enseignant du lycée (équivalent à la fin du secondaire québécois) se considère plutôt comme un «prof de lettres». Serait-ce que l'enseignement de la littérature n'aurait pas sa place au $1^{\text {er }}$ cycle puisque les apprentissages essentiels (savoirlire, savoir-écrire) n'y seraient pas encore maitrisés ? Les enseignants interrogés ont affirmé que le principal défi, surtout au $1^{\text {er }}$ cycle, est celui de développer des compétences en lecture et en écriture, car leurs élèves sont encore en train de les construire. Par exemple, Sophie affirme poursuivre des objectifs différents entre les classes régulières et celles d'un programme d'élite, le Programme d'éducation internationale (PÉI), car «au régulier, il y en a ça leur prend deux minutes pour lire une page, alors ils finissent la page et ils ne se souviennent pas de ce qu'ils ont lu à la page précédente». Les enseignants composent avec la réalité de leur classe, souvent marquée d'une hétérogénéité sociale, culturelle 
et cognitive, particulièrement dans les classes du régulier, bien plus nombreuses que celles des programmes d'élite. Pour les enseignants, l'enjeu majeur est de développer des compétences à lire et de tenter, à travers cet objectif, de donner le gout de lire à leurs élèves, ce qui ressort de façon prononcée au $1^{\text {er }}$ cycle.

Cette tension entre les deux cycles apparait également dans les modes de lecture littéraire ${ }^{12}$ privilégiés. Notre analyse fait ressortir qu'au $1^{\text {er }}$ cycle, l'enseignement de la «littérature» se concentre sur des activités de réception centrées sur un mode de lecture littéraire participative (on demande aux élèves de partager leurs réactions, d'expliquer en quoi ils s'identifient au protagoniste, etc.). Au $2^{\mathrm{e}}$ cycle, les activités de réception tendent vers une lecture littéraire plus distanciée en privilégiant une lecture critique. Il semble donc qu'au $1^{\text {er }}$ cycle, la littérature soit moins utilisée comme objet d'apprentissage que comme objet de plaisir et de découverte de soi, alors qu'au $2^{\mathrm{e}}$ cycle, on chercherait à lui donner plus d'espace pour développer des habiletés d'analyse et de réflexion critique. Rien ne semble avoir changé depuis plus de 45 ans :

[...] aujourd'hui comme hier, indépendamment de tous les essais de rénovation pédagogique, la part du littéraire va croissant au fur et à mesure qu'on avance dans la scolarité. Aux cycles inférieurs prime l'étude de la langue tandis qu'aux cycles supérieurs l'étude du littéraire l'emporte. (Simard, 1997b, p. 206)

Nos résultats confirment qu'on enseignerait moins la littérature en tant qu'objet que le savoir-lire et le savoir-écrire, compétences langagières développées avec des textes littéraires ou non littéraires.

\subsection{Rôle de la littérature dans le développement des savoirs : développer des compétences langagières et culturelles}

Que peut-on apprendre avec la littérature? Pour 8 des 10 enseignants, la principale fonction épistémique de la littérature est le développement des compétences langagières décrit sous trois angles. Le premier concerne les compétences en lecture, c'est-à-dire travailler des stratégies de lecture (surtout au $1^{\text {er }}$ cycle) afin de bonifier la compréhension et les interprétations (surtout au $2^{\mathrm{e}}$ cycle), soit comme support

12. Selon Dufays (2006), trois modes de lecture littéraire coexistent : la participation (lecture plus affective), la distanciation (lecture plus intellectualisée) et l'oscillation entre la participation et la distanciation. 
pour travailler l'écriture, par exemple en analysant les caractéristiques d'un genre afin de le faire produire dans une activité d'écriture créative, soit comme support pour faire produire des analyses littéraires pour travailler l'argumentation (surtout en $5^{\mathrm{e}}$ secondaire, soit à la fin du $2^{\mathrm{e}}$ cycle). Le deuxième repose sur l'idée de développer une meilleure compréhension de la langue, par l'étude du vocabulaire recherché et les structures de phrases modèles. Le troisième, évoqué pour le $2^{\mathrm{e}}$ cycle, renvoie à la formation de l'esprit critique par les représentations du monde qu'offrent les œuvres et qui poussent à réfléchir sur la vie ou à connaitre et comprendre des réalités culturelles ou sociohistoriques.

Les deux premiers angles, dominants, sont motivés par les prescriptions où, au moins depuis le programme de 1995, on demande notamment d'enseigner les stratégies de lecture au $1^{\text {er }}$ cycle, choix porté par le courant de la psychologie cognitive de l'enseignement de la lecture (Giasson, 1990), très populaire dans les années 1990, et la production d'un texte d'opinion argumenté à la fin du $2^{\mathrm{e}}$ cycle, afin que les élèves réussissent l'épreuve d'écriture de certification nationale du secondaire. La littérature devient donc un outil pour exemplifier un objet prescrit par les programmes (stratégies de lecture, texte argumentatif, grammaire). Le troisième angle semble évoquer les principes guidant les derniers programmes dans lesquels on insiste pour que les élèves «multiplie[nt] et diversifie[nt] les expériences culturelles afin de se constituer de nouveaux repères pour comprendre le monde» (MÉLS, 2009, p. 3).

Il est clair qu'avec les programmes chargés et imprécis, et les classes hétérogènes du régulier, les choix sont priorisés en fonction des connaissances et des compétences des enseignants, des évaluations certificatives nationales ou régionales, du matériel disponible et des compétences des élèves. Entre les classes plutôt homogènes du PÉI et les classes hétérogènes du régulier, les enseignants planifient leurs séquences d'enseignement dans un système à deux vitesses où, dans les classes trop nombreuses du régulier, souvent composées d'élèves plus faibles, on priorise l'enseignement de la grammaire et le travail sur les stratégies de lecture comme l'ont montré Chartrand et Lord (2013). En revanche, au PÉI (et autres filières d'élite) s'ajoute un deuxième programme à celui du MÉLS, dans lequel on prescrit d'enseigner l'histoire de la littérature, en ciblant des courants littéraires à étudier pour chaque année du secondaire. Les didacticiens de la littérature défendent l'idée, souvent idéaliste, que si les enseignants étaient formés pour construire des séquences d'enseignement organisées autour d'œuvres intégrales 
où les caractéristiques génériques et culturelles seraient étudiées, et si ces séquences s'articulaient étroitement au travail sur la langue et sur la littérature, la littérature serait peut-être moins instrumentalisée et plus enseignée en tant qu'objet (Biard \& Denis, 1993; Dufays, 2006; Langlade, 2002; Legros, 2000 ; Veck, 1998). Or, la réalité de l'enseignement en classe de français est qu'on y travaille surtout le savoir-lire et savoir-écrire, comme le rappelle Sophie : «Je réalise que quand je cherche des séquences en enseignement de la littérature, je ne pense qu'au [PÉI]. J'ai l'impression que, dans mon groupe de régulier, on en fait beaucoup moins sinon presque pas du tout».

\section{Conclusion}

Si les analyses descriptives n'apportent pas de résultats fulgurants ni même très étonnants, elles présentent néanmoins souvent l'intérêt d'étayer des observations, des intuitions, des idées reçues, même quand elles portent sur des échantillons très limités. Tel est l'objectif de notre contribution, et plus largement de RELIT. L'analyse a fait ressortir des différences entre les pratiques d'enseignement aux deux cycles du secondaire, autant à propos du choix des corpus enseignés que des finalités d'enseignement de la littérature. Elle permet de dégager des conclusions qui font écho à d'autres recherches sur l'enseignement des textes littéraires (De Beaudrap, 2004 ; Dezutter, 2007), en plus de mettre en lumière des facteurs structurant le RL d'enseignants de français. Nous confirmons ainsi les tendances dégagées par ces recherches, en plus d'identifier trois autres facteurs qui influencent clairement les conceptions des enseignants et le rôle qu'ils donnent à la littérature dans le développement des savoirs : les pratiques personnelles de la littérature, la formation initiale et le contexte d'enseignement.

Plus les enseignants multiplient leurs pratiques de la littérature (par exemple, Sophie écrit de la poésie au quotidien depuis des années, Gilles lit plusieurs œuvres par semaine), plus ils ont une conception complexe de la littérature. En outre, notre analyse montre que la formation initiale a un impact important sur leurs conceptions et pratiques de la littérature : on voit un rapport étroit entre le temps d'études (nombre de crédits en études littéraires) et le type d'études (formation initiale en études littéraires ou études supérieures menant à un diplôme de maitrise) et leur RL. Il ressort que moins les sujets ont reçu une formation littéraire élaborée (notamment Sébastien et Julie), moins ils convoquent de critères pour décrire la littérature et moins ils ont des pratiques de littérature dans 
le plan personnel de leur RL; plus leur formation semble spécialisée (Fabrice, Sonia, Sophie, Gilles), plus ils ont de pratiques personnelles de littérature et plus ils se rapprochent d'une conception systémique de la littérature. Par ailleurs, fait troublant, aucun sujet de l'étude n'a affirmé avoir bénéficié de formation continue en didactique du français, mais ils sont nombreux à avoir exprimé le besoin d'en recevoir afin de se sentir plus compétents, constante observée dans la recherche ÉLEF (Chartrand \& Lord, 2013). Il faut dire que la formation continue des enseignants au Québec n'est pas obligatoire et qu'elle est très limitée en raison du manque de financement des écoles et des commissions scolaires : la formation continue est donc laissée à la volonté et souvent aux frais des enseignants.

De plus, l'analyse montre à quel point les conditions matérielles, sociales et scolaires du travail enseignant ont des effets sur leurs pratiques. Les didacticiens de la littérature proposent que les enseignants mettent en place des séquences didactiques organisées autour d'une œuvre littéraire intégrale, mais, sans une politique d'accès aux livres, les enseignants doivent composer avec les œuvres disponibles dans l'école, tenir compte des compétences langagières de leurs élèves et des défis qui s'y rattachent, selon qu'ils enseignent dans des milieux privilégiés ou populaires.

Autre constante, chaque œuvre littéraire devant être lue par les élèves fait l'objet d'une évaluation, quand bien même elle n'a pas été travaillée en classe; les enseignants disent évaluer systématiquement la lecture des œuvres littéraires de leurs élèves. Les systèmes scolaires sont de plus en plus centrés sur l'évaluation des apprentissages (Fourez, 2003), reléguant au second rang les conditions d'apprentissage et la formation des enseignants. Ces derniers sont obsédés par les évaluations qui orientent de plus en plus les savoirs enseignés (tous les enseignants de $5^{\mathrm{e}}$ secondaire travaillent le texte d'opinion, certains même l'intègrent dans leur séquence d'enseignement de la littérature, car l'épreuve ministérielle de fin de secondaire concerne ce genre).

Enfin, on peut se poser la question suivante : les auteurs des programmes et les didacticiens de la littérature ont-ils tous pleinement conscience des conditions concrètes d'exercice du travail d'enseignant dans des systèmes scolaires de plus en plus stratifiés socialement et culturellement, inégalement pourvus matériellement et en partie dévoyés par l'obligation de résultat (Mauduit, 2003) ? Nous avons cherché à relever - au moins partiellement - ce défi en montrant le lien entre conditions d'exercice du travail et conceptions et pratiques déclarées 
d'enseignement de la littérature. Cependant, cet article présente certaines limites, notamment à l'égard des pratiques enseignantes effectives. Celles-ci seront d'ailleurs l'objet d'une prochaine contribution (phase II de RELIT) qui nous permettra de mieux décrire les effets du RL sur les pratiques effectives pour comprendre ce qui s'enseigne sous l'appellation «littérature ».

\section{RÉFÉRENCES BIBLIOGRAPHIQUES}

Aron, Paul \& Viala, Alain. (2005). L'enseignement littéraire. Paris : PUF. BarRÉ-DE-MiniaC, Christine. (2000). Le rapport à l'écriture : aspects théoriques et didactiques. Villeneuve-d'Ascq : Septentrion.

Biard, Jacqueline \& Denis, Frédérique. (1993). Didactique du texte littéraire. Paris : Nathan.

Blaser, Christiane. (2007). Fonction épistémique de l'écrit : pratiques et conceptions d'enseignants de sciences et d'histoire du secondaire (Thèse de doctorat, Université Laval, Québec).

Blaser, Christiane. (2009). Le synopsis : un outil méthodologique pour comprendre la pratique enseignante. Nouveaux cahiers de la recherche en éducation, 12(1), 117-129.

BOURDIER, Pierre. (1992). Les Règles de l'art. Genèse et structure du champ littéraire. Paris : Seuil.

Charlot, Bernard, Bautier, Élisabeth \& Rochex, Jean-Yves. (1992). École et savoir dans les banlieues... et ailleurs. Paris : Armand Colin.

Chartrand, Suzanne-G. \& Blaser, Christiane. (2008). Le rapport à l'écrit : un outil pour enseigner de l'école à l'université. Namur: Presses de l'Université de Namur.

Chartrand, Suzanne-G. \& Lord, Marie-Andrée. (2010). Pratiques déclarées d'enseignement de l'écriture et de la lecture. Québec français, 156, 30-31.

Chartrand, Suzanne-G. \& Lord, Marie-Andrée. (2013). L'enseignement de la grammaire et de l'écriture au secondaire québécois : principaux résultats d'une recherche descriptive. Revue suisse des Sciences de l'éducation, 35(3), 515-539.

Daunay, Bertrand. (2007). État des recherches en didactique de la littérature. Revue française de pédagogie, 159, 139-189.

De BeAudrap, Anne-Raymonde (dir.). (2004). Images de la littérature et de son enseignement. Nantes : CNDP-CRDPP.

Dezutter, Olivier, Larivière, Isabelle, Bergeron, Marie-Douce \& MorisSETTE, Carl. (2007). Les pratiques déclarées des enseignants québécois dans la sélection et l'exploitation des œuvres complètes inscrites au 
programme de lecture des élèves. Dans É. Falardeau et coll., La didactique du français. Les voies actuelles de la recherche (p. 83-100). Québec : PUL.

DufaYs, Jean-Louis. (2006). La lecture littéraire: des «pratiques du terrain» aux modèles théoriques. Revue de linguistique et de didactique des langues, 33, 79-101.

Dufays, Jean-Louis, Lisse, Michel \& Meurée, Christophe. (2009). Théorie de la littérature : une introduction. Louvain-la-Neuve, Bel. : AcademiaBruylant.

Émery-Bruneau, Judith. (2010). Le rapport à la lecture littéraire. Des pratiques et des conceptions de sujets-lecteurs en formation à l'enseignement du français à des intentions didactiques (Thèse de doctorat, Université Laval, Québec).

ÉMERY-BRunEAU, Judith. (2011). La dimension sociale du rapport à la lecture littéraire d'étudiants en enseignement : un indice du développement de l'identité professionnelle. Revue canadienne des sciences de l'éducation, 34(2), 34-52.

ÉMERY-BRUNEAU, Judith. (2012). La dimension subjective du rapport à la lecture littéraire de futurs enseignants de français. Dans J.-L. Dumortier, J. Van Beveren \& D. Vrydaghs (dir.), Curriculum et progression en français (p. 111-132). Namur : Presses universitaires de Namur.

FALARDEAU, Érick \& Simard, Denis. (2011). L'étude du rapport à la culture dans les pratiques enseignantes : le synopsis comme outil de réduction et d'organisation des données. Recherches qualitatives, 30(2), 96-121.

FouREz, Gérard. (2003). Évaluation, relations pédagogiques, rôle de l'erreur. La Revue nouvelle, 5-6, 75-89.

Giasson, Jocelyne. (1990). La compréhension en lecture. Boucherville : Gaétan Morin.

Lebrun, Monique (dir.). (2004). Les pratiques de lecture des adolescents québécois. Montréal : Multimonde.

LANGLADE, Gérard. (2002). Lire des æeuvres intégrales au collège et au lycée. Toulouse : Delagrave/CRDP Midi-Pyrénées.

L'ÉCUYER, René. (1990). Méthodologie de l'analyse développementale des contenus. Méthode GPS et concept de soi. Québec : PUQ.

Legros, Georges. (2000). Quelle littérature enseigner? Dans M.-J. Fourtanier \& G. Langlade (dir.), Enseigner la littérature (p. 19-30). Toulouse : Delagrave/CRDP Midi-Pyrénées.

Mauduit, Jean-Bernard. (2003). Le territoire de l'enseignant. Esquisse d'une critique de la raison enseignante. Paris : Klincksieck.

MÉQ. (2005). Programme de formation de l'école québécoise. Enseignement secondaire, premier cycle. Québec : Gouvernement du Québec. 
MÉLS. (2009). Programme de formation de l'école québécoise. Enseignement secondaire, deuxième cycle. Québec : Gouvernement du Québec.

Schneuwly, Bernard \& Dolz, Joaquim (dir.). (2009). Des objets enseignés en classe de français. Le travail de l'enseignant sur la rédaction de textes argumentatifs et sur la subordonnée relative. Rennes: PUR.

SÉnéchal, Kathlenne \& Chartrand, Suzanne-G. (2012). Représentations et pratiques de l'enseignement de l'oral en classe de français : changements et constantes depuis 25 ans. Dans R. Bergeron \& G. PlessisBélair (dir.), Représentations, analyses et descriptions du français oral, de son utilisation et de son enseignement au primaire, au secondaire et à l'université (p. 185-199). Montréal : Peisaj.

SIMARD, Claude. (1997a). Éléments de didactique du français langue première. Montréal : ERPI.

SIMARD, Claude. (1997b). Dynamique du rapport entre didactique de la langue et didactique de la littérature. Dans M. Noël-Gaudreault (dir.), Didactique de la littérature. Bilan et perspectives (p. 197-209). Montréal : Nuit blanche.

Simard, Claude, Dufays, Jean-Louis, Dolz, Joaquim \& Garcia-Debanc, Claudine. (2010). Didactique du français langue première. Bruxelles : De Boeck.

VECK, Bernard. (1998). Euvres intégrales et projet de lecture. Paris : Éd. Bertrand-Lacoste. 
ANNEXES

Tableau 1. - Grille d'analyse du plan personnel du rapport à la littérature $(\mathrm{RL})$.

\begin{tabular}{|c|c|c|c|}
\hline \multicolumn{4}{|c|}{ PLAN PERSONNEL (PP) du RL } \\
\hline $\begin{array}{l}\text { Dimension } \\
\text { subjective }\end{array}$ & $\begin{array}{l}\text { Dimension } \\
\text { épistémique }\end{array}$ & $\begin{array}{l}\text { Dimension } \\
\text { sociale }\end{array}$ & $\begin{array}{c}\text { Dimension } \\
\text { praxéologique }\end{array}$ \\
\hline $\begin{array}{l}\text { PPDSu-1 : } \\
\text { Représentations de } \\
\text { la littérature }\end{array}$ & $\begin{array}{l}\text { PPDE-1 : Nature } \\
\text { des savoirs / savoir- } \\
\text { faire convoqués }\end{array}$ & $\begin{array}{l}\text { PPDSo-1 : Rôle des } \\
\text { autres et réseaux } \\
\text { littéraires de } \\
\text { socialisation }\end{array}$ & $\begin{array}{l}\text { PPDP-1 : Types } \\
\text { de production ou } \\
\text { objet de réception } \\
\text { (quoi ?) }\end{array}$ \\
\hline $\begin{array}{l}\text { PPDSu-2 : } \\
\text { Projets littéraires } \\
\text { personnels }\end{array}$ & $\begin{array}{l}\text { PPDE-2 : Rôle des } \\
\text { savoirs / savoir-faire } \\
\text { en littérature }\end{array}$ & $\begin{array}{l}\text { PPDSo-2 : Rôle de } \\
\text { la littérature dans } \\
\text { les interactions } \\
\text { sociales }\end{array}$ & $\begin{array}{l}\text { PPDP-2 : Lieux de } \\
\text { production ou de } \\
\text { réception (où?) }\end{array}$ \\
\hline $\begin{array}{l}\text { PPDSu-3 : } \\
\text { Réflexivité par } \\
\text { rapport à la } \\
\text { littérature }\end{array}$ & $\begin{array}{l}\text { PPDE-3 : } \\
\text { Conceptions des } \\
\text { savoirs / savoir-faire }\end{array}$ & $\begin{array}{l}\text { PPDSo-3 : Rôle de } \\
\text { l'école en littérature }\end{array}$ & $\begin{array}{l}\text { PPDP-3 : Contexte } \\
\text { de production ou de } \\
\text { réception (quand?) }\end{array}$ \\
\hline $\begin{array}{l}\text { PPDSu-4 : Aspects } \\
\text { axiologiques de la } \\
\text { littérature }\end{array}$ & $\begin{array}{l}\text { PPDE-4 : Rôle de } \\
\text { la littérature dans le } \\
\text { développement des } \\
\text { savoirs/savoir-faire }\end{array}$ & $\begin{array}{l}\text { PPDSo-4 : Rôle } \\
\text { des enseignants } \\
\text { préuniversitaires }\end{array}$ & $\begin{array}{l}\text { PPDP-4 : Finalités } \\
\text { ou objectifs de } \\
\text { production ou } \\
\text { de réception } \\
\text { (pourquoi?) }\end{array}$ \\
\hline $\begin{array}{l}\text { PPDSu-5 : Aspects } \\
\text { psychoaffectifs de } \\
\text { la littérature }\end{array}$ & $\begin{array}{l}\text { PPDE-5 : Critères } \\
\text { de sélection ou } \\
\text { valeur symbo- } \\
\text { lique des savoirs / } \\
\text { savoir-faire }\end{array}$ & $\begin{array}{l}\text { PPDSo-5 : Rôle de } \\
\text { la formation initiale }\end{array}$ & $\begin{array}{l}\text { PPDP-5 : Mode } \\
\text { de production } \\
\text { ou de réception } \\
\text { (comment?) }\end{array}$ \\
\hline \multirow[t]{2}{*}{$\begin{array}{l}\text { PPDSu-6 : Critères } \\
\text { de littérarité des } \\
\text { textes }\end{array}$} & & $\begin{array}{l}\text { PPDSo-6 : Rôle } \\
\text { de la formation } \\
\text { continue }\end{array}$ & $\begin{array}{l}\text { PPDP-6 : } \\
\text { Temps et fréquence } \\
\text { de production } \\
\text { ou de réception } \\
\text { (combien?) }\end{array}$ \\
\hline & & & $\begin{array}{l}\text { PPDP-7 : Moyens } \\
\text { didactiques ou } \\
\text { pédagogiques }\end{array}$ \\
\hline
\end{tabular}




\section{Tableau 2. - Grille d'analyse du plan didactique du rapport à la littérature $(\mathrm{RL})$.}

\begin{tabular}{|c|c|c|c|}
\hline \multicolumn{4}{|c|}{ PLAN DIDACTIQUE (PD) du RL } \\
\hline $\begin{array}{l}\text { Dimension } \\
\text { subjective }\end{array}$ & $\begin{array}{l}\text { Dimension } \\
\text { épistémique }\end{array}$ & $\begin{array}{l}\text { Dimension } \\
\text { sociale }\end{array}$ & $\begin{array}{c}\text { Dimension } \\
\text { praxéologique }\end{array}$ \\
\hline $\begin{array}{l}\text { PDDSu-1 : } \\
\text { Représentations de } \\
\text { la littérature des } \\
\text { élèves }\end{array}$ & $\begin{array}{l}\text { PDDE-1 : Nature } \\
\text { des savoirs / savoir- } \\
\text { faire convoqués en } \\
\text { classe }\end{array}$ & $\begin{array}{l}\text { PDDSo-1 : } \\
\text { Rôle des autres et } \\
\text { réseaux littéraires } \\
\text { des élèves }\end{array}$ & $\begin{array}{l}\text { PDDP-1 : Types } \\
\text { de production des } \\
\text { élèves ou objet de } \\
\text { réception (quoi?) }\end{array}$ \\
\hline $\begin{array}{l}\text { PDDSu-2 : } \\
\text { Projets littéraires } \\
\text { personnels des } \\
\text { élèves }\end{array}$ & $\begin{array}{l}\text { PDDE-2 : Rôle des } \\
\text { savoirs / savoir-faire } \\
\text { en littérature en } \\
\text { classe }\end{array}$ & $\begin{array}{l}\text { PDDSo-2 : Rôle de } \\
\text { la littérature dans } \\
\text { les interactions } \\
\text { sociales des élèves }\end{array}$ & $\begin{array}{l}\text { PDDP-2 : Lieux de } \\
\text { production ou de } \\
\text { réception des élèves } \\
\text { (où?) }\end{array}$ \\
\hline $\begin{array}{l}\text { PDDSu-3 : } \\
\text { Réflexivité des } \\
\text { élèves par rapport à } \\
\text { la littérature }\end{array}$ & $\begin{array}{l}\text { PDDE-3 : } \\
\text { Conceptions des } \\
\text { savoirs / savoir-faire } \\
\text { des élèves }\end{array}$ & $\begin{array}{l}\text { PDDSo-3 : Rôle de } \\
\text { l'école en littérature } \\
\text { pour les élèves }\end{array}$ & $\begin{array}{l}\text { PDDP-3 : Contexte } \\
\text { de production ou de } \\
\text { réception des élèves } \\
\text { (quand?) }\end{array}$ \\
\hline $\begin{array}{l}\text { PDDSu-4 : Aspects } \\
\text { axiologiques des } \\
\text { élèves par rapport à } \\
\text { la littérature }\end{array}$ & $\begin{array}{l}\text { PDDE-4 : Rôle de } \\
\text { la littérature dans le } \\
\text { développement des } \\
\text { savoirs / savoir-faire } \\
\text { des élèves }\end{array}$ & $\begin{array}{l}\text { PDDSo-4 : Rôle } \\
\text { des enseignants } \\
\text { du primaire ou du } \\
\text { secondaire }\end{array}$ & $\begin{array}{l}\text { PDDP-4 : Finalités } \\
\text { ou objectifs de } \\
\text { production ou de } \\
\text { réception des élèves } \\
\text { (pourquoi?) }\end{array}$ \\
\hline $\begin{array}{l}\text { PDDSu-5 : Aspects } \\
\text { psychoaffectifs des } \\
\text { élèves par rapport à } \\
\text { la littérature }\end{array}$ & $\begin{array}{l}\text { PDDE-5 : Critères } \\
\text { de sélection ou } \\
\text { valeur symbolique } \\
\text { des savoirs / savoir- } \\
\text { faire des élèves }\end{array}$ & & $\begin{array}{l}\text { PDDP-5 : Mode de } \\
\text { production ou de } \\
\text { réception des élèves } \\
\text { (comment?) }\end{array}$ \\
\hline \multirow[t]{2}{*}{$\begin{array}{l}\text { PDDSu-6 : Critères } \\
\text { des élèves quant } \\
\text { à la littérarité des } \\
\text { textes }\end{array}$} & & & $\begin{array}{l}\text { PDDP-6 : } \\
\text { Temps et fréquence } \\
\text { de production ou de } \\
\text { réception des élèves } \\
\text { (combien?) }\end{array}$ \\
\hline & & & $\begin{array}{l}\text { PDDP-7 : Moyens } \\
\text { didactiques ou } \\
\text { pédagogiques }\end{array}$ \\
\hline
\end{tabular}

